\title{
Buprenorphine Microinduction: Logistical Barriers and the Need for Convergent Evidence
}

\author{
Joao P. De Aquino $0^{1,2}$ (D) Suprit Parida ${ }^{1,2} \cdot$ Mehmet Sofuoglu ${ }^{1,2}$ \\ Accepted: 26 May 2021 / Published online: 9 June 2021 \\ (c) The Author(s), under exclusive licence to Springer Nature Switzerland AG 2021
}

\section{Dear Editor,}

We thank Dr. Accurso for his interesting report on the clinical use of buprenorphine microinduction [1]. We agree that there are logistical barriers that contribute to the difficulty in implementing microinduction, especially in primary-care settings [2, 3]. First, healthcare professionals must write complex orders, with progressive increases in the dose of buprenorphine, as well as frequent changes in its schedule of administration. Second, to adhere to the microinduction protocol, patients are often required to break sublingual tablets of buprenorphinewhich may lead to uneven dosing - and to follow complex instructions. Together, these barriers can make both patients and clinicians less inclined to adopt microinduction as a viable approach - their removal, thus, may increase the success rates of induction onto buprenorphine.

Electronic medical record (EMR) order sets are common and may promote efficient patient care. Since the development of buprenorphine microinduction as a method is still in early stages, an ongoing evaluation of EMR order sets-and their adjustment based on real-world patient outcomes-is warranted [4]. Especially if guided by data from randomized, controlled clinical trials, such EMR order sets may provide a platform with which to titrate buprenorphine microinduction approaches to produce optimal clinical outcomes.

We also commend Dr. Accurso's leadership in collaborating with local pharmacies to dispense ready-made microinduction blister packs. Convergent lines of evidence

This comment refers to the article available at: https://doi.org/10. 1007/s40261-021-01032-7.

Joao P. De Aquino

joao.deaquino@yale.edu

1 VA Connecticut Healthcare System, 950 Campbell Avenue (151D), Building 36/116A4, West Haven, CT 06516, USA

2 Department of Psychiatry, Yale University School of Medicine, 300 George Street, New Haven, CT 06511, USA indicate that such initiatives may enhance medication adherence, including for populations among whom substance use and psychiatric disorders are highly prevalent $[5,6]$. Whether such initiatives impact the adherence or response to buprenorphine microinduction remain to be determined.

In summary, by using a combination of approaches to the problem of how to best start buprenorphine treatment-with multi-pronged evidence from mechanistic studies, observational data, and clinical trials - it may be possible to develop tailored clinical strategies to prevent precipitated withdrawal, thereby reducing its impact on delivering effective pharmacotherapy for opioid use disorder in various clinical settings.

\section{Declarations}

Funding No funding was received to prepare this letter.

Conflict of interest The authors have no conflicts of interest to report.

\section{References}

1. Accurso AJ. Blister-packing of $2 \mathrm{mg}$ buprenorphine monoproduct as a patient-centered method of microdosing for buprenorphine induction. Clin Drug Investig. 2021. https://doi.org/10.1007/ s40261-021-01048-z

2. Parida S, De Aquino JP, Sofuoglu M. High potency synthetic opioids: curbing the third wave of the opioid crisis. Neurosci Biobehav Rev. 2019;106:9.

3. De Aquino JP, Parida S, Sofuoglu M. The pharmacology of buprenorphine microinduction for opioid use disorder. Clin Drug Investig. 2021;2021:1-12.

4. McGreevey JD III. Order sets in electronic health records: principles of good practice. Chest. 2013;143(1):228-35.

5. Gutierrez PM, Wortzel HS, Forster JE, Leitner RA, Hostetter TA, Brenner LA. Blister packaging medication increases treatment adherence in psychiatric patients. J Psychiatr Pract. 2017;23(5):320-7.

6. Cunningham EB, Amin J, Feld JJ, Bruneau J, Dalgard O, Powis $\mathrm{J}$, et al. Adherence to sofosbuvir and velpatasvir among people with chronic HCV infection and recent injection drug use: the SIMPLIFY study. Int J Drug Policy. 2018;62:14-23. 